Recepción: 20 / 04 / 2017

Aceptación: 20 / 05 / 2017

Publicación: 15 / 06 / 2017

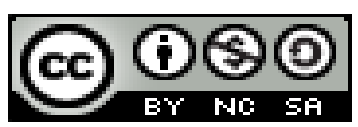

Ciencias de la Educación

Artículo de Investigación

\title{
Uso de técnicas activas en el aprendizaje del área de Lengua y Literatura para el desarrollo de habilidades y destrezas
}

Use of active techniques in the learning of the area of Language and Literature for the development of skills and abilities

\section{Usando técnicas de aprendizagem activa na área de Língua e Literatura para o desenvolvimento de competências e habilidades}

Janet E. Pazmiño-Ramírez ${ }^{1}$ janetpazmino@hotmail.com

Silvia A. Medina-Anchundia ${ }^{\mathrm{II}}$ silvia.medinaa@ug.edu.ec

Juan C. Ramos-Romero III juanka.ramos@gmail.com

Correspondencia: janetpazmino@ hotmail.com

I. Magister en Diseño Curricular; Profesora Especialización Educación Primaria, Profesora de Segunda Enseñanza en la Especialización de Comercio y Administración, Licenciada en Ciencias de la Educación Especialización Educación Primaria, Universidad de Guayaquil, Guayaquil, Ecuador.

II. Magister en Administración de Empresas; Ingeniera Comercial; Universidad de Guayaquil, Guayaquil, Ecuador.

III. Magister en Docencia y Gerencia en Educación Superior; Ingeniero en Sistemas Computacionales, Universidad Agraria del Ecuador, Guayaquil, Guayas, Ecuador. 


\section{Resumen}

El presente trabajo está inmerso en la realidad educativa donde fue investigada en el Centro de Educación Básico $\mathrm{N}^{\circ} 508$ "Dr. Clemente Yerovi Indaburu" está ubicado en Lomas de la Florida Ciudadela Socio Vivienda de la Parroquia Tarqui, Cantón Guayaquil, Provincia del Guayas, el mismo que trata de Las Técnicas Activas y su incidencia en el Proceso de Enseñanza- Aprendizaje del Área de Lengua y Literatura. Por lo tanto, su tratamiento didáctico y metodológico está estrechamente ligado al trabajo del docente y a la aplicación de Técnicas que permitan a los estudiantes fomentar, cultivar y desarrollar una serie de habilidades y destrezas que le son útiles para participar efectivamente en el proceso de enseñanza aprendizaje. La estructura del trabajo cuenta con: el planteamiento del tema, problema, objetivos e hipótesis, de la misma manera considera el tratamiento de contenidos teóricos que fundamentan la investigación, el marco metodológico de este trabajo constituye el escenario técnico operacional, donde se menciona el conjunto de métodos, técnicas y recursos que se emplearon en el proceso de la tesis, se complementa con la investigación de campo desarrollada en la institución educativa, misma, que arrojó resultados que apoyan la elaboración de una Guía Didáctica con técnicas activas para ser utilizadas por los docentes. Por otra parte, la investigación aborda temas de interés, especialmente relacionados con la importancia del área de Lengua y Literatura y con la propuesta, que da a conocer una serie de técnicas para utilizarse y mejorar el nivel de aprendizajes significativos de los estudiantes del Sexto Año de Educación Básica. Para llevar a cabo la aplicación de la propuesta se implementará una capacitación en el conocimiento de aplicación de Técnicas Activas que conlleven a obtener un mejor rendimiento. Al finalizar el trabajo se encuentran una serie de recomendaciones y conclusiones que ayudarán al trabajo docente.

Palabras clave: Enseñanzas de aprendizaje; proceso de enseñanza - aprendizaje; habilidades y destrezas; lengua y literatura; técnicas de aprendizaje. 


\section{Abstract}

This paper is immersed in the educational reality which was investigated, Basic Education Center No. 508 " Dr. Indaburu Yerovi Clemente " is located in Lomas de la Florida Citadel Parish Housing Partner Tarquinia, Canton Guayaquil, Guayas Province, is the same as Active Techniques and its impact on the teaching-learning process of the Department of Language and Literature. Therefore, didactic and methodological treatment is closely linked to the work of teaching and the application of techniques that allow students to encourage, nurture and develop a series of abilities and skills that are useful to participate effectively in the teaching-learning process. The structure of the work includes: the approach to the topic, problem, objectives and hypothesis, in the same way considered the treatment of underlying theoretical research, the methodological framework of this work is the technical-operational stage, which mentions the set of methods, techniques and resources used in the process of the thesis, is complemented by field research developed in the educational institution itself, which yielded results that support the development of an educational guide with active techniques to be used by teachers . Moreover, the research addresses issues of interest, especially related to the importance of language arts area and the proposal, which discloses a number of techniques to use and improve the level of meaningful learning for students of Sixth Year Basic Education. To carry out the implementation of the proposal will be implemented training in the application of technical knowledge of Active that lead to better performance. After work is a series of recommendations and conclusions that will help the teaching

Key words: Learning skills; teaching - learning process; skills and abilities; language and literature; learning techniques. 


\section{Resumo}

O presente trabalho está imerso na realidade educativa, onde foi investigado, Centro de Educação Básica "Dr. Clemente Yerovi Indaburu "está localizado em Lomas da Flórida Ciudadela Socio Vivienda da Paróquia Tarqui, Cantão Guayaquil, Provincia del Guayas, o mesmo que trata de Las Técnicas Activas e incidência no Processo de Enseñanza- Aprendizagem do Área de Lengua e Literatura . Por lo tanto, o tratamento didáctico e metodológico está estreitamente ligado ao trabalho de docente e a aplicação de Técnicas que permitam aos estudantes fomentar, cultivar e desenvolver uma série de habilidades e produtos que le servem para participar efetivamente no processo de ensino . A estrutura do trabalho conta com: o planteamento do tema, problema, objetivos e hipóteses, da mesma forma, considerando o tratamento de conteúdos teóricos que fundamentam a pesquisa, o marco metodológico do trabalho constituído pelo escenario técnico operacional, onde se menciona o conjunto De métodos e recursos que se empregaram no processo da prova, se complementa com a investigação de campo desenvolvida na institução educativa, mesma, que arrojó resultados que favorecem a elaboração de uma Guia Didáctica con técnicas ativas para ser us por los Docentes. Por outra parte, a investigação aborda temas de interesse, especialmente relacionados com a importância da área de Lengua e Literatura e a proposta, que conhece uma série de técnicas para melhorar e melhorar o nível de aprendizagens dos estudantes do sexo grado De Educação Básica. Para levar a cabo a aplicação da proposta de implementação de treinamento no conhecimento de aplicação de Técnicas Activas que conlleven a obter um melhor desempenho. Al finalizar o trabalho, encontre uma série de recomendações e conclusões que ajudem no trabalho docente

Palavras chave: Aprendendo lições; ensino - aprendizagem; competências e habilidades; língua e Literatura; técnicas de aprendizagem. 


\section{Introducción.}

En el transcurso de la historia, la educación ha constituido un factor importante en el desarrollo de la sociedad, y mundialmente se le consagran grandes recursos, aunque no todos los países le dan la prioridad requerida a pesar de reconocer su importancia.

La educación es la idea angular para el desarrollo de una nación y para la formación del ser humano, por ello el Ministerio de Educación Ecuatoriano se vio en la necesidad de hacer una revisión a nuestro currículo Educativo el cual incluye, como una de sus políticas, el mejoramiento de la calidad de la educación. En 1996 se oficializó un nuevo currículo para Educación General Básica fundamentado en el desarrollo de destrezas y la aplicación de ejes transversales que recibió el nombre de "Reforma Curricular de la Educación Básica".

En 2007 la Dirección Nacional de Currículo realizó un estudio a nivel nacional que permitió determinar el grado de aplicación de la Reforma Curricular de la Educación Básica en las aulas, determinando los logros y dificultades, tanto técnicas como didácticas.

Cada una de las áreas del currículo nacional tiene características específicas en cuanto a contenidos, objetivos, estrategias metodológicas y evaluación, sin embargo, convergen en la formación integral de los estudiantes. Formación que inicia desde edades muy tempranas y que está sujeta al tratamiento de contenidos utilizados como medios para desarrollar habilidades y destrezas de carácter específico.

Se debe señalar que el director y los docentes que laboran en la descrita institución, me han proporcionado las debidas facilidades que permiten realizar la investigación con mayor rapidez y eficacia. 
En consecuencia, el objetivo de la investigación se cumplirá de manera exitosa, contribuyendo al mejoramiento de la práctica pedagógica dentro y fuera del aula, pero más que nada, al desarrollo de las habilidades y destrezas de los estudiantes del Sexto Año de Educación Básica.

Por lo anteriormente mencionado es recomendable que las instituciones educativas cambien el modelo educativo que ha venido llevando durante los últimos años, por el bien de los estudiantes e implanten técnicas en donde todos los estudiantes se integren a la clase para que puedan desarrollar sus habilidades y destrezas de tal manera que se cree un ambiente agradable en donde logren desenvolverse con creatividad, criticidad y flexibilidad y no tener estudiantes receptivos.

\section{Materiales y métodos.}

Los métodos utilizados en la investigación son los siguientes:

Método empírico. - Se utilizó este método porque su aporte en el proceso de la investigación permitió obtener el resultado fundamental de la misma por medio de la observación, la experimentación, la entrevista, la encuesta que dio facilidad al estudio de los fenómenos observables, confirmar la hipótesis planteada además del análisis y comprensión de las teorías investigadas.

Método estadístico. - Este método ayudó a determinar la muestra y la población; a estudiar, tabular y analizar los datos de los resultados que se obtuvieron de las encuestas y poder a establecer las generalizaciones apropiadas a partir de ello. 
Método teórico. - Este método ayudó a construir y desarrollar la teoría científica necesaria, para abordar problemas de la ciencia permitiendo profundizar en el conocimiento de las regularidades y cualidades esenciales del objetivo de estudio, además de su análisis y síntesis.

Método deductivo. - Este método en el trabajo de investigación permitió realizar un análisis científico general en el proceso investigativo, partiendo de los hechos encontrados en la problemática existente la institución que es el aspecto, general de los hechos.

Método inductivo. - Este método se lo utilizó cuando se empezó a investigar la teoría científica, estructurar los objetivos, formular la hipótesis, las demostraciones, plantear la propuesta que es la parte fundamental del trabajo de la investigación; permitió realizar el estudio de las conexiones necesarias con el objeto de investigación, relaciones de causalidad, entre otros.

Método lógico. - Permitió interrelacionar el pasado y el presente, además, de validar su evolución, dando de esta manera un argumento valedero para la presente tesis.

Método bibliográfico. - A través del cual se pudo realizar la investigación bibliográfica inmediata, utilizando en la biblioteca libros, folletos, internet, para poder determinar el problema de investigación; manejándose así, datos específicos en algunos libros; que corresponden a las respuestas de las cuestiones realizadas en este trabajo.

\section{Tipos de investigación}

Descriptiva: Es descriptiva porque a través de lo observado se describió los acontecimientos tal como estaba sucediendo en la realidad; lo que permitió hacer un estudio profundo para desarrollar este trabajo de investigación. 
Janet E. Pazmiño-Ramírez; Silvia A. Medina-Anchundia; Juan C. Ramos-Romero

Campo o Laboratorio: La presente investigación es de campo, porque se la aplica en el lugar de los hechos, basándose en la recolección primaria de los datos, que provienen de las entrevistas, encuestas y observaciones. Es decir, se obtuvo la información directamente de la realidad en la que se encuentra.

Aplicada. - La intención de este trabajo fue identificar el problema para darle solución al mismo, de tal manera que será aplicada en el Centro de Educación Básica No 508 “Dr. Clemente Yerovi Indaburu” de la ciudad de Guayaquil, que es la institución donde se detectó el problema.

Técnicas e instrumentos para la obtención de datos Encuestas

Para la aplicación de este trabajo de investigación se utilizará: Técnicas: Entrevista y Instrumentos: Cuestionario, guía de preguntas estructuradas.

Diseño por la dimensión temporal:

Esta investigación es transversal porque se la realizará en un tiempo determinad, un año lectivo.

Universo y muestra

El universo de esta investigación lo constituyen los estudiantes del Sexto Año "A” director y docentes de la institución; se aplicará entrevista al director de la institución y encuesta a los docentes y a los estudiantes del Sexto Año Paralelo "A" del Centro de Educación Básica, tal como se detalla a continuación: 
Población:

\begin{tabular}{|l|c|c|}
\hline \multicolumn{1}{|c|}{ GRUPOS } & POBLACIÓN & \% \\
\hline Director & 1 & 1 \\
\hline Profesores & 12 & 21 \\
\hline Estudiantes & 45 & 18 \\
\hline Total de la Muestra & 58 & 100 \\
\hline
\end{tabular}

Datos: Centro de Educación Básica $N^{\circ} 508$ “Dr. Clemente Yerovi Indaburu”

Muestra:

Como los estudiantes del Sexto Año "A" del Centro de Educación Básica N 508 "Dr.

Clemente Yerovi Indaburu” son 45 se tomará a todos para la investigación.

\section{Resultados.}

RESUTADO DE LAS ENCUESTAS A DOCENTES DEL CENTRO DE EDUCACIÓN BÁSICA No 508 “DR. CLEMENTE YEROVI INDABURU

1.- ¿En clases de Lengua y Literatura desarrolla técnicas que estimulan la actividad en sus estudiantes?

\section{CUADRO $\mathbf{N}^{\circ} 1$}

\begin{tabular}{|l|c|l|l|}
\hline $\mathbf{N}^{\circ}$ & INDICADORES & FRECUENCIA & $\%$ \\
\hline $\mathbf{1}$ & Siempre & 0 & 0 \\
\hline $\mathbf{2}$ & A veces & 5 & 42 \\
\hline $\mathbf{3}$ & Nunca & 7 & 58 \\
\hline & TOTAL & 12 & 100 \\
\hline
\end{tabular}

Datos: Centro de Educación Básica $N^{\circ} 508$ "Dr. Clemente Yerovi Indaburu” 
Janet E. Pazmiño-Ramírez; Silvia A. Medina-Anchundia; Juan C. Ramos-Romero

\section{GRÁFICO $\mathbf{N}^{\circ} 1$}

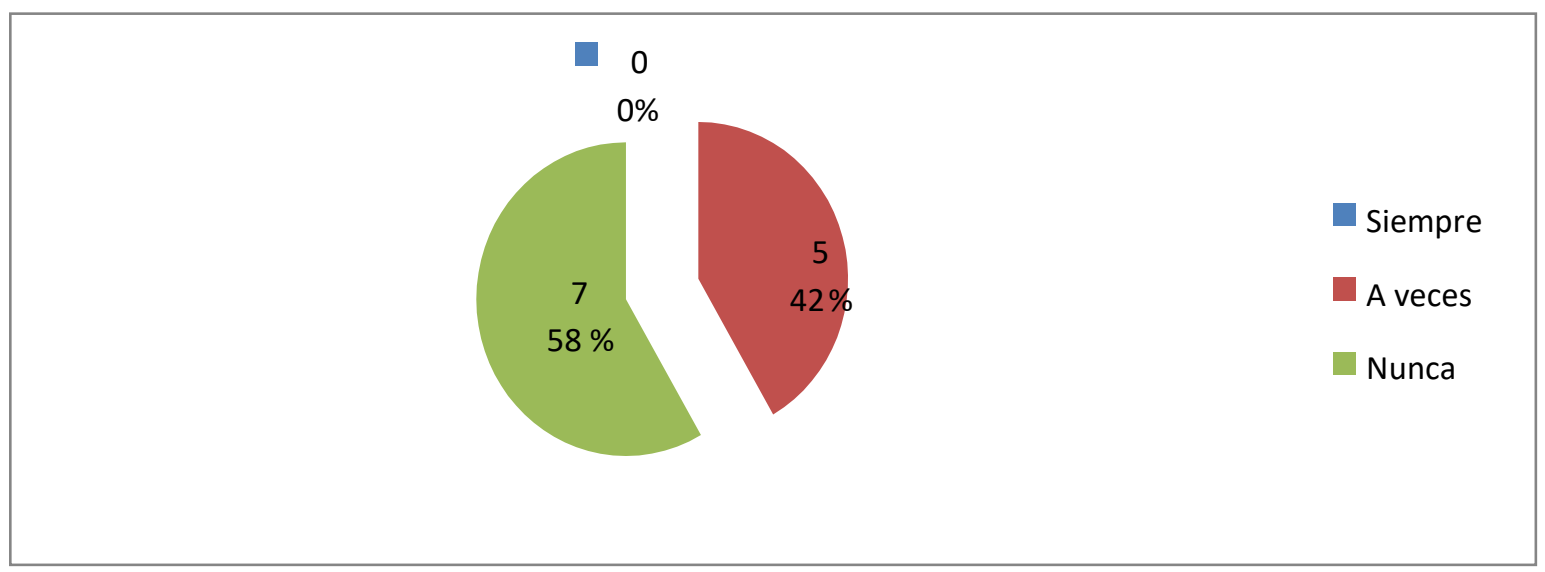

Datos: Centro de Educación Básica N 508 “Dr. Clemente Yerovi Indaburu”

Análisis e Interpretación

Encuestados los docentes sobre si utilizan alguna técnica en las clases de Lengua y Literatura que estimulen la actividad en los estudiantes, el 58\% manifiestan que nunca, el $42 \%$ casi siempre; ninguno contestó nunca.

Con estos resultados se establece que los maestros no utilizan las técnicas activas en el proceso aprendizaje de los estudiantes, pese a que así lo manifiesta la reforma curricular; porque con su aplicación en el aula de clase se promueve la participación activa de los estudiantes dentro de la misma. 
2.- ¿Para los trabajos en el aula usted realiza un diagnóstico de los conocimientos previos, y aplica alguna técnica para mejorar el proceso aprendizaje?

\section{CUADRO $\mathbf{N}^{\circ} 2$}

\begin{tabular}{|c|l|c|c|}
\hline $\mathbf{N}^{\circ}$ & INDICADORES & FRECUENCIA & $\boldsymbol{\%}$ \\
\hline $\mathbf{1}$ & Siempre & 1 & 8 \\
\hline $\mathbf{2}$ & A veces & 5 & 42 \\
\hline $\mathbf{3}$ & Nunca & 6 & 50 \\
\hline & TOTAL & 16 & 100 \\
\hline
\end{tabular}

Datos: Centro de Educación Básica $N^{\circ} 508$ "Dr. Clemente Yerovi Indaburu”

\section{GRÁFICO $\mathbf{N}^{\circ} 2$}

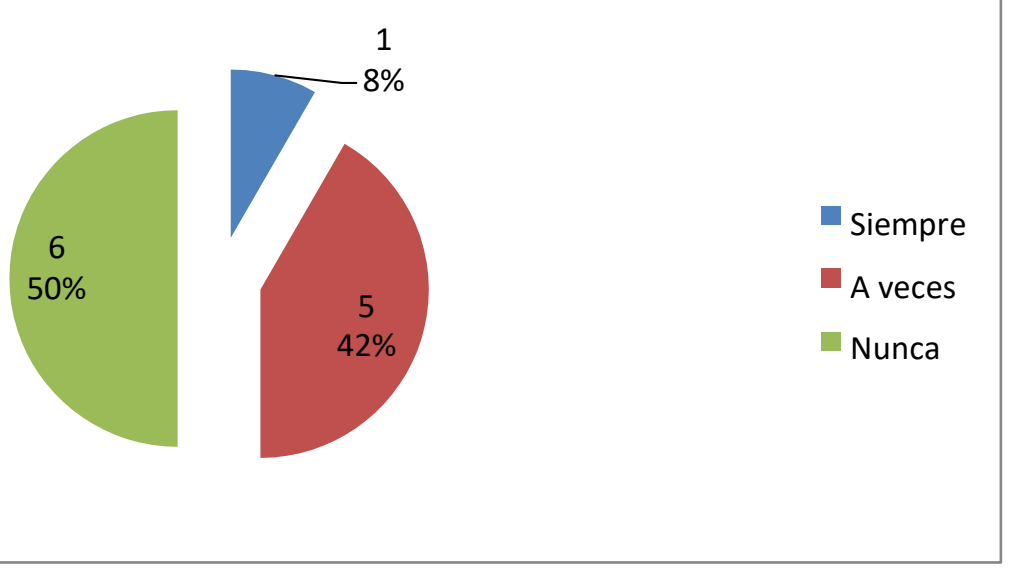

Datos: Centro de Educación Básica $N^{\circ} 508$ "Dr. Clemente Yerovi Indaburu”

Análisis e Interpretación

En esta pregunta sobre los trabajos en el aula usted realiza un diagnóstico de los conocimientos previos, y aplica alguna técnica para mejorar el proceso aprendizaje, el 50\% contestó nunca, el $42 \%$ casi siempre y el $8 \%$ siempre. 
Janet E. Pazmiño-Ramírez; Silvia A. Medina-Anchundia; Juan C. Ramos-Romero

Una del base fundamental al iniciar una clase, es el diagnóstico para poder darnos cuenta como docentes cuáles son los conocimientos, experiencias, errores, de los estudiantes y poder llenar esas expectativas, pero siempre tomando en cuenta las actitudes y necesidades del grupo.

\section{3.- Sus estudiantes sienten agrado por el área de Lengua y Literatura?}

\section{CUADRO $\mathbf{N}^{\circ} 3$}

\begin{tabular}{|l|l|c|c|}
\hline $\mathbf{N}^{\circ}$ & INDICADORES & FRECUENCIA & $\boldsymbol{\%}$ \\
\hline $\mathbf{1}$ & Siempre & 3 & 25 \\
\hline $\mathbf{2}$ & A veces & 4 & 33 \\
\hline $\mathbf{3}$ & Nunca & 5 & 42 \\
\hline & TOTAL & 12 & 100 \\
\hline
\end{tabular}

Datos: Centro de Educación Básica $N^{\circ} 508$ “Dr. Clemente Yerovi Indaburu”

\section{GRÁFICO No 3}

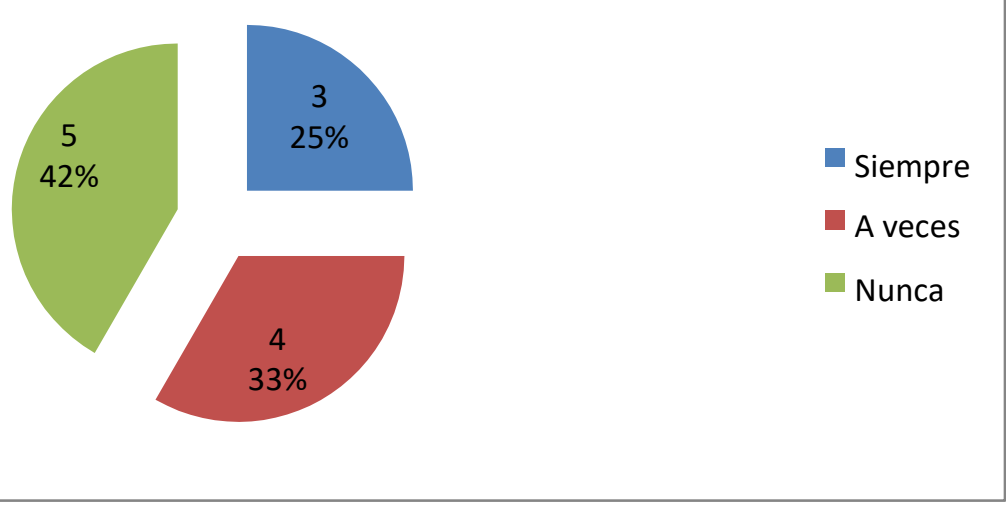

Datos: Centro de Educación Básica $N^{\circ} 508$ “Dr. Clemente Yerovi Indaburu”

Análisis e Interpretación

El $42 \%$ de los docentes contestaron que a sus estudiantes no les agrada el área de Lengua y Literatura, el $33 \%$ a veces y el $25 \%$ siempre. 
El área de Lengua y Literatura es una de las áreas básicas, cuya enseñanza se basa esencialmente en la importante función que cumple la misma en el desarrollo de la capacidad comunicativa, y, en consecuencia, para el desarrollo del pensamiento. Asimismo, es fundamental la enseñanza de la literatura la enseñanza de la Literatura atendiendo que esta contribuye para la formación estética de las personas, por lo tanto, los docentes deben poner mucho énfasis al momento de enseñar esta área y tratar de hacerla más agradable para los estudiantes y esto se debe realizar aplicando técnicas activas en su enseñanza.

4.- Considera usted que el uso adecuado de técnicas activas en el aprendizaje del área de Lengua y Literatura promueven un rendimiento excelente en los estudiantes?

\section{CUADRO $\mathbf{N}^{\circ} 4$}

\begin{tabular}{|l|l|c|c|}
\hline $\mathbf{N}^{\circ}$ & \multicolumn{1}{|c|}{ INDICADORES } & FRECUENCIA & $\%$ \\
\hline $\mathbf{1}$ & Siempre & 9 & 75 \\
\hline $\mathbf{2}$ & A veces & 3 & 25 \\
\hline $\mathbf{3}$ & Nunca & 0 & 0 \\
\hline & TOTAL & 12 & 100 \\
\hline
\end{tabular}

Datos: Centro de Educación Básica $N^{\circ} 508$ “Dr. Clemente Yerovi Indaburu” 
Janet E. Pazmiño-Ramírez; Silvia A. Medina-Anchundia; Juan C. Ramos-Romero

\section{GRÁFICO N ${ }^{\circ} 4$}

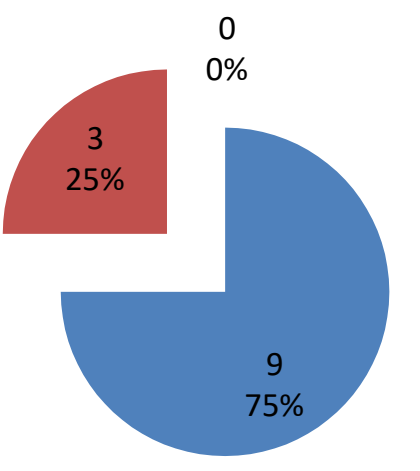

Siempre

A veces

Nunca

\section{Datos: Centro de Educación Básica N 508 “Dr. Clemente Yerovi Indaburu”}

\section{Análisis e Interpretación}

El $75 \%$ de los encuestados considera que el uso adecuado de técnicas activas en el aprendizaje del área de Lengua y Literatura promueven un rendimiento excelente en los estudiantes, el $25 \%$ considera que A veces y nadie opina que Nunca.

Como hemos manifestado en la parte científica que los usos de técnicas activas en el aprendizaje de todas las áreas originan un aprendizaje grato, puesto que las activas invitan al estudiante a la participación en la clase, y más aún en el área de Lengua y Literatura porque es un área de gran interés para los estudiantes porque la lengua representa una herramienta fundamental para la interacción social. 


\section{5.- Comparte experiencias con sus compañeros docentes?}

\section{CUADRO $\mathbf{N}^{\circ} 5$}

\begin{tabular}{|c|l|c|c|}
\hline $\mathbf{N}^{\circ}$ & INDICADORES & FRECUENCIA & \% \\
\hline $\mathbf{1}$ & Siempre & 2 & 17 \\
\hline $\mathbf{2}$ & A veces & 3 & 25 \\
\hline $\mathbf{3}$ & Nunca & 7 & 58 \\
\hline & TOTAL & 12 & 100 \\
\hline
\end{tabular}

Datos: Centro de Educación Básica $N^{\circ} 508$ "Dr. Clemente Yerovi Indaburu”

\section{GRÁFICO $\mathbf{N}^{\circ} 5$}
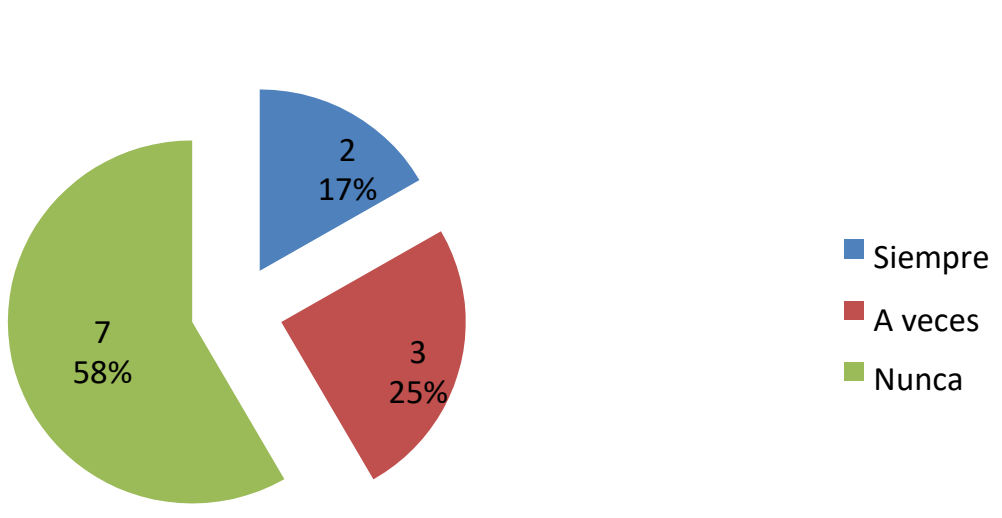

\section{Datos: Centro de Educación Básica N 508 "Dr. Clemente Yerovi Indaburu”}

Análisis e Interpretación

De acuerdo con las respuestas de esta pregunta se determina que el $58 \%$ de los docentes nunca comparten experiencias con sus compañeros docentes; el 25\% a veces: el $17 \%$ siempre.

Como podemos observar en estos resultados los docentes no son comunicativos entre compañeros, siendo la acción comunicativa de suma importancia en el quehacer educativo, y necesario en este caso que ellos cambien de actitud porque de lo contrario básicamente afecta como 
Janet E. Pazmiño-Ramírez; Silvia A. Medina-Anchundia; Juan C. Ramos-Romero

es lógico, la relación y derivan en una disminución notable de la eficacia docente; y por lo tanto repercute en el aprendizaje de los estudiantes

6.- ¿Se apoya de bibliografía adicional para la enseñanza área de Lengua y Literatura? CUADRO $\mathbf{N}^{\circ} 6$

\begin{tabular}{|l|l|c|c|}
\hline $\mathbf{N}^{\circ}$ & \multicolumn{1}{|c|}{ INDICADORES } & FRECUENCIA & $\%$ \\
\hline $\mathbf{1}$ & Siempre & 0 & 0 \\
\hline $\mathbf{2}$ & A veces & 3 & 25 \\
\hline $\mathbf{3}$ & Nunca & 9 & 75 \\
\hline & TOTAL & 12 & 100 \\
\hline
\end{tabular}

Datos: Centro de Educación Básica N 508 “Dr. Clemente Yerovi Indaburu”

\section{GRÁFICO N ${ }^{\circ} 6$}

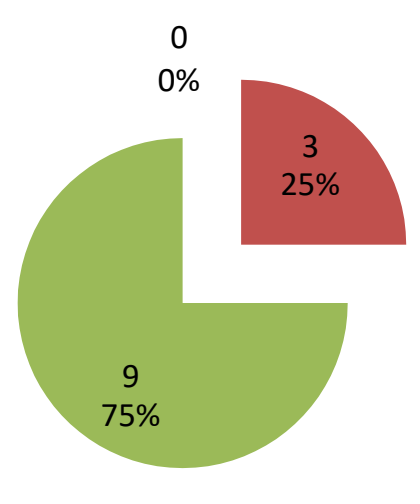

Datos: Centro de Educación Básica $N^{\circ} 508$ “Dr. Clemente Yerovi Indaburu” 
Análisis e Interpretación

De acuerdo a los resultados de los encuestados en esta pregunta si se apoya de bibliografía adicional para la enseñanza área de Lengua y Literatura el 75\% respondieron que nunca; el $25 \%$ a veces, nadie contestó siempre.

Siendo las técnicas un proceso para lograr participación en los estudiantes y lograr un aprendizaje significativo, se hace necesario que los docentes busquen bibliografías que contengan dichas técnicas activas para aplicarlas en la enseñanza del área de Lengua y Literatura y lograr en los estudiantes aprendizaje significativo.

7.- ¿Estaría dispuesto a trabajar con un recurso de aprendizaje que integre técnicas activas que contribuyan al aprendizaje de Lengua y Literatura?

\section{CUADRO $\mathbf{N}^{\circ} 7$}

\begin{tabular}{|l|l|l|l|}
\hline $\mathbf{N}^{\circ}$ & \multicolumn{1}{|c|}{ INDICADORES } & FRECUENCIA & $\%$ \\
\hline $\mathbf{1}$ & Siempre & 12 & 100 \\
\hline $\mathbf{2}$ & A veces & 0 & 0 \\
\hline $\mathbf{3}$ & Nunca & 0 & 0 \\
\hline & TOTAL & 12 & 100 \\
\hline
\end{tabular}

Datos: Centro de Educación Básica $N^{\circ} 508$ “Dr. Clemente Yerovi Indaburu” 
Janet E. Pazmiño-Ramírez; Silvia A. Medina-Anchundia; Juan C. Ramos-Romero

\section{GRÁFICO N ${ }^{\circ}$}

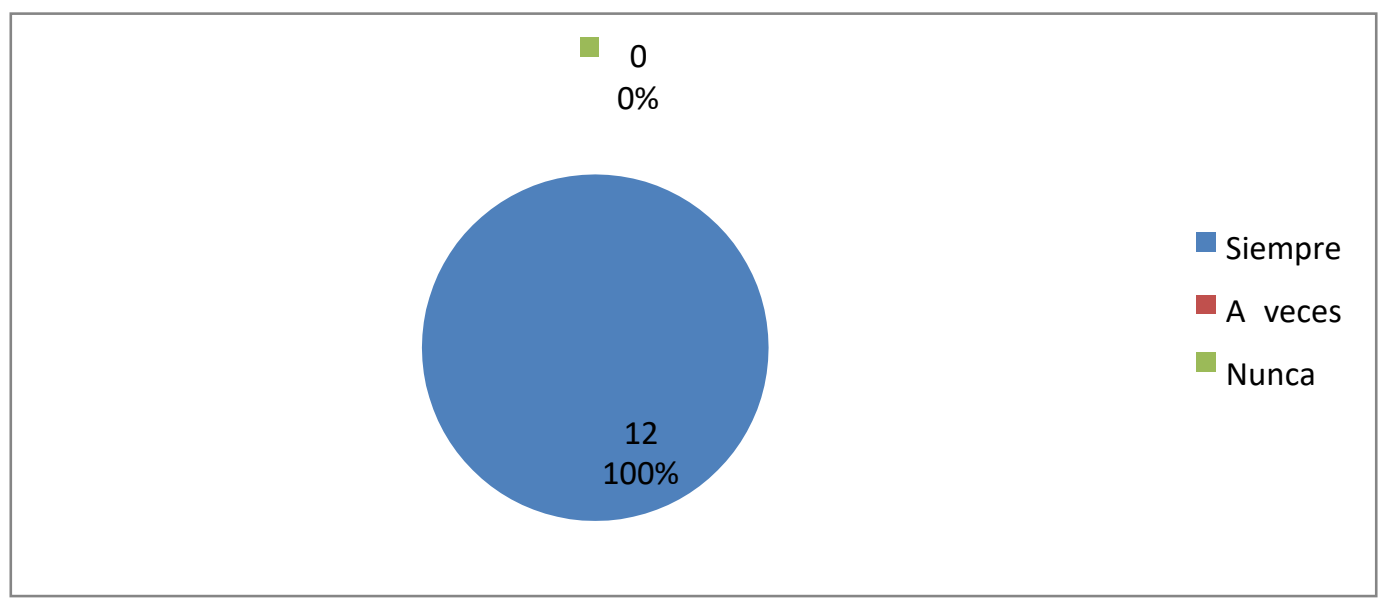

Datos: Centro de Educación Básica $N^{\circ} 508$ “Dr. Clemente Yerovi Indaburu”

Análisis e Interpretación

En los resultados obtenidos en esta pregunta qué si estaría dispuesto a trabajar con un recurso de aprendizaje que integre técnicas activas que contribuyan al aprendizaje de Lengua y Literatura el 100\% respondió siempre, nadie respondió a veces o nunca.

Los objetivos primordiales del cambio educativo están en lograr en los estudiantes un desarrollo amplio y profundo del conocimiento, además del desarrollo de habilidades, destrezas, actitudes y valores, por lo que el docente debe rediseñar el proceso de enseñanza y en este desarrollo es que se sustenta el cambio del modelo educativo; siendo así está obligado a buscar recursos de aprendizaje que contengan técnicas activas que contribuyan al bienestar de los estudiantes. 
8.- ¿Creen necesario que los docentes tengan una guía con técnicas activas de aprendizaje?

\section{CUADRO $\mathbf{N}^{\circ} 8$}

\begin{tabular}{|l|l|c|c|}
\hline $\mathbf{N}^{\circ}$ & \multicolumn{1}{|c|}{ INDICADORES } & FRECUENCIA & $\%$ \\
\hline $\mathbf{1}$ & Siempre & 12 & 100 \\
\hline $\mathbf{2}$ & A veces & 0 & 0 \\
\hline $\mathbf{3}$ & Nunca & 0 & 0 \\
\hline & TOTAL & 12 & 100 \\
\hline
\end{tabular}

Datos: Centro de Educación Básica $N^{\circ} 508$ "Dr. Clemente Yerovi Indaburu”

\section{GRÁFICO $\mathbf{N}^{\circ} 8$}

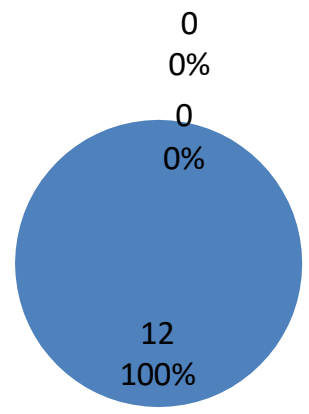

Siempre

A veces

Nunca

Datos: Centro de Educación Básica $N^{\circ} 508$ “Dr. Clemente Yerovi Indaburu”

Análisis e Interpretación

De los resultados obtenidos en esta pregunta si es necesario que los docentes tengan una guía con técnicas activas de aprendizaje el $100 \%$ de los docentes contestaron que siempre, nadie contestó a veces o nunca.

Podemos darnos cuenta que la actualización de los docentes en cuanto a técnicas activas es importante debido a que estamos inmersos en cambios de orden educativos y culturales, en este sentido los docentes deben estar capacitados, pues en la metodología de la enseñanza surge el 
modelo constructivista que se centra en los procesos internos de los estudiantes, concibiéndolos como participantes dinámicos y recomienda la utilización de técnicas activas de enseñanza; porque busca preparar estudiantes autónomos, creativos, con capacidad crítica, de resolver problemas de autorregularse y sobre todo de aprender a aprender. Por lo que se hace necesaria la propuesta de elaborar una guía didáctica con técnicas activas que favorezcan el aprendizaje del área de Lengua y Literatura en los estudiantes.

\section{RESUTADO DE LAS ENCUESTAS A LOS ESTUDIANTES DEL}

\section{SEXTO AÑO PARALELO “A” DEL CENTRO DE EDUCACIÓN BÁSICA}

\section{No 508 "DR. CLEMENTE YEROVI INDABURU}

\section{1.- ¿La maestra inicia las clases con una dinámica?}

\section{CUADRO $\mathbf{N}^{\circ} 9$}

\begin{tabular}{|l|l|c|c|}
\hline $\mathbf{N}^{\circ}$ & INDICADORES & FRECUENCIA & $\boldsymbol{\%}$ \\
\hline $\mathbf{1}$ & SI & 10 & 22 \\
\hline $\mathbf{2}$ & NO & 30 & 67 \\
\hline $\mathbf{3}$ & TAL VEZ & 5 & 11 \\
\hline & TOTAL & 45 & 100 \\
\hline
\end{tabular}

Datos: Centro de Educación Básica $N^{\circ} 508$ “Dr. Clemente Yerovi Indaburu” 


\section{GRÁFICO $\mathbf{N}^{\circ} 9$}

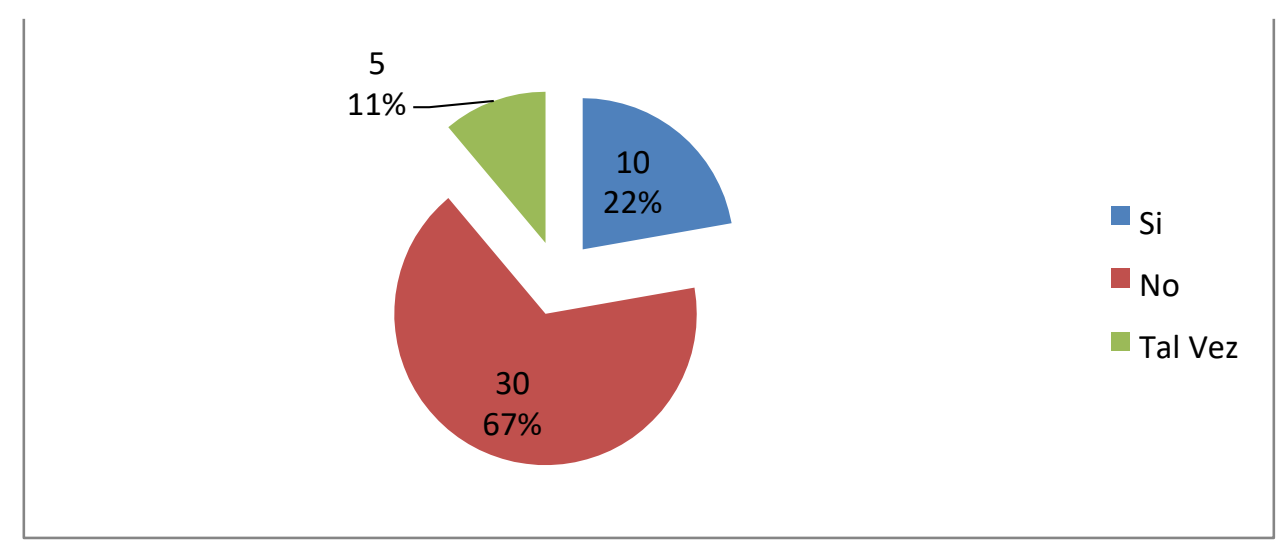

Datos: Centro de Educación Básica No 508 “Dr. Clemente Yerovi Indaburu”

Análisis e Interpretación

De los 45 estudiantes encuestados el $67 \%$ contestaron que su maestra no inicia las clases con dinámicas; el $22 \%$ si y el $11 \%$ tal vez.

Con este resultado podemos deducir que la motivación es importante en el aula de clase porque de ella depende inicialmente las necesidades y los impulsos de los estudiantes, puestos que estos elementos originan la voluntad de aprender en general y concentran la voluntad de ellos, concibiéndose en un ambiente agradable, donde son tomados en cuenta como persona que sienten piensan y desean y dirigen sus energías para aprender. 
Janet E. Pazmiño-Ramírez; Silvia A. Medina-Anchundia; Juan C. Ramos-Romero

\section{2.- ¿El trabajo que desarrolla la maestra es activo?}

\section{CUADRO $\mathbf{N}^{\circ} 10$}

\begin{tabular}{|l|l|c|c|}
\hline $\mathbf{N}^{\circ}$ & INDICADORES & FRECUENCIA & \% \\
\hline $\mathbf{1}$ & SI & 10 & 22 \\
\hline $\mathbf{2}$ & NO & 27 & 13 \\
\hline $\mathbf{3}$ & TAL VEZ & 8 & 65 \\
\hline & TOTAL & 45 & 100 \\
\hline
\end{tabular}

Datos: Centro de Educación Básica $N^{\circ} 508$ “Dr. Clemente Yerovi Indaburu"

\section{GRÁFICO N ${ }^{\circ} 10$}

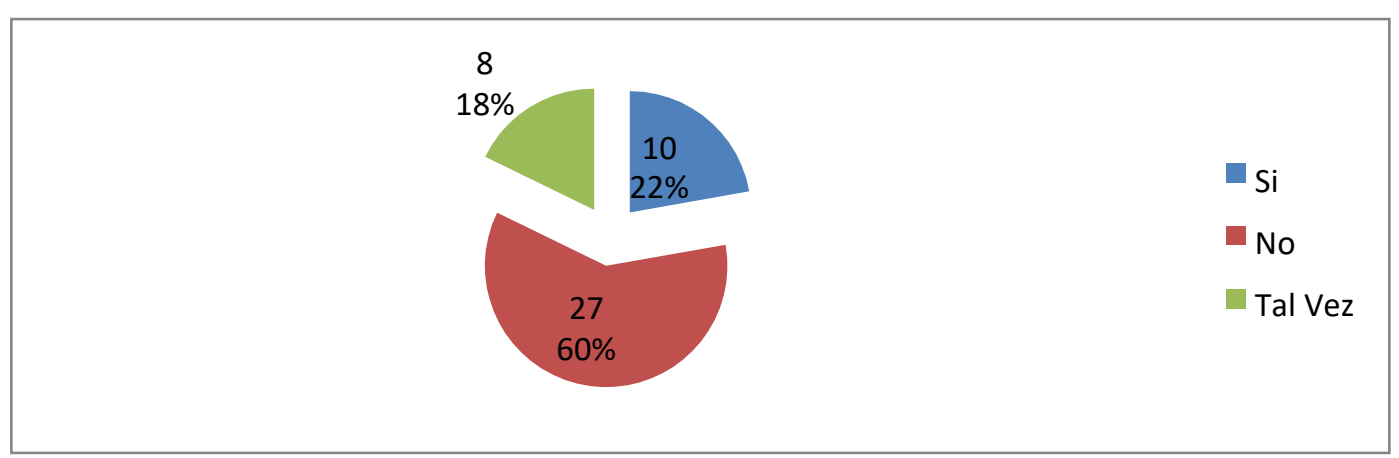

Datos: Centro de Educación Básica $N^{\circ} 508$ “Dr. Clemente Yerovi Indaburu”

Análisis e Interpretación

El resultado que se obtuvo en esta interrogativa sobre si el trabajo que desarrolla la maestra es activo el $60 \%$ contestaron que no, el $22 \%$ que si y el $18 \%$ tal vez.

Con estos resultados de los estudiantes encuestados se confirma la necesidad de aplicar este trabajo en el Centro de educación Básica "Dr. Clemente Yerovi Indaburu”, en donde los docentes deben de cambiar esa forma tradicional de impartir la enseñanza y empezar a emplear las técnicas activas de enseñanza, donde el estudiante el responsable de la construcción de su propio aprendizaje. 
3.- ¿Le gustaría que su maestra imparta las clases con juegos, cantos, dramas, crucigramas, etc.?

\section{CUADRO $\mathbf{N}^{\circ} 11$}

\begin{tabular}{|l|l|c|c|}
\hline $\mathbf{N}^{\circ}$ & INDICADORES & FRECUENCIA & $\boldsymbol{\%}$ \\
\hline $\mathbf{1}$ & SI & 38 & 84 \\
\hline $\mathbf{2}$ & NO & 0 & 0 \\
\hline $\mathbf{3}$ & TAL VEZ & 7 & 18 \\
\hline & TOTAL & 45 & 100 \\
\hline
\end{tabular}

Datos: Centro de Educación Básica No 508 “Dr. Clemente Yerovi Indaburu”

\section{GRÁFICO No 11}
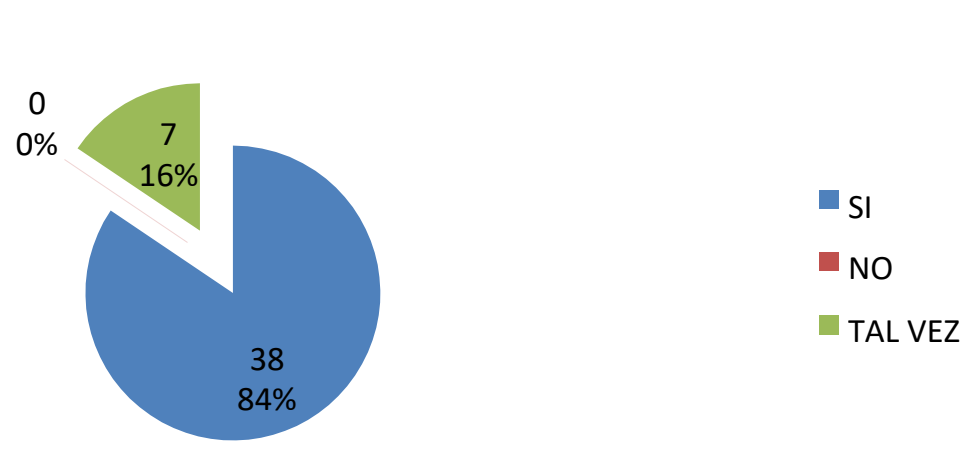

Datos: Centro de Educación Básica No 508 “Dr. Clemente Yerovi Indaburu”

Análisis e Interpretación

El $84 \%$ de los estudiantes investigados contestaron que, si les gustaría que su maestra imparta las clases con juegos, cantos, dramas, crucigramas, etc.; el $16 \%$ contestó que tal vez y nadie respondió que no.

La base científica de este trabajo se apoya en la utilización de técnicas activas de aprendizaje; por lo tanto, el aprendizaje debe ser activo, basado en el estudiante, y este solo se lo puede adquirir a través de la implicación, motivación, y trabajo constante del estudiante. Por lo 
Janet E. Pazmiño-Ramírez; Silvia A. Medina-Anchundia; Juan C. Ramos-Romero

tanto, se requiere por parte del docente una nueva manera d conducir la clase y asumir un nuevo papel; es decir ser el guía de sus estudiantes y hacer que ellos aprendan.

4.- ¿Le ayuda a aprender Lengua y Literatura trabajando con organizadores gráficos?

\section{CUADRO $\mathbf{N}^{\circ} 12$}

\begin{tabular}{|l|l|c|c|}
\hline $\mathbf{N}^{\circ}$ & INDICADORES & FRECUENCIA & $\mathbf{\%}$ \\
\hline $\mathbf{1}$ & SI & 40 & 89 \\
\hline $\mathbf{2}$ & NO & 0 & 0 \\
\hline $\mathbf{3}$ & TAL VEZ & 5 & 11 \\
\hline & TOTAL & 45 & 100 \\
\hline
\end{tabular}

Datos: Centro de Educación Básica $N^{\circ} 508$ “Dr. Clemente Yerovi Indaburu”

\section{GRÁFICO N 12}

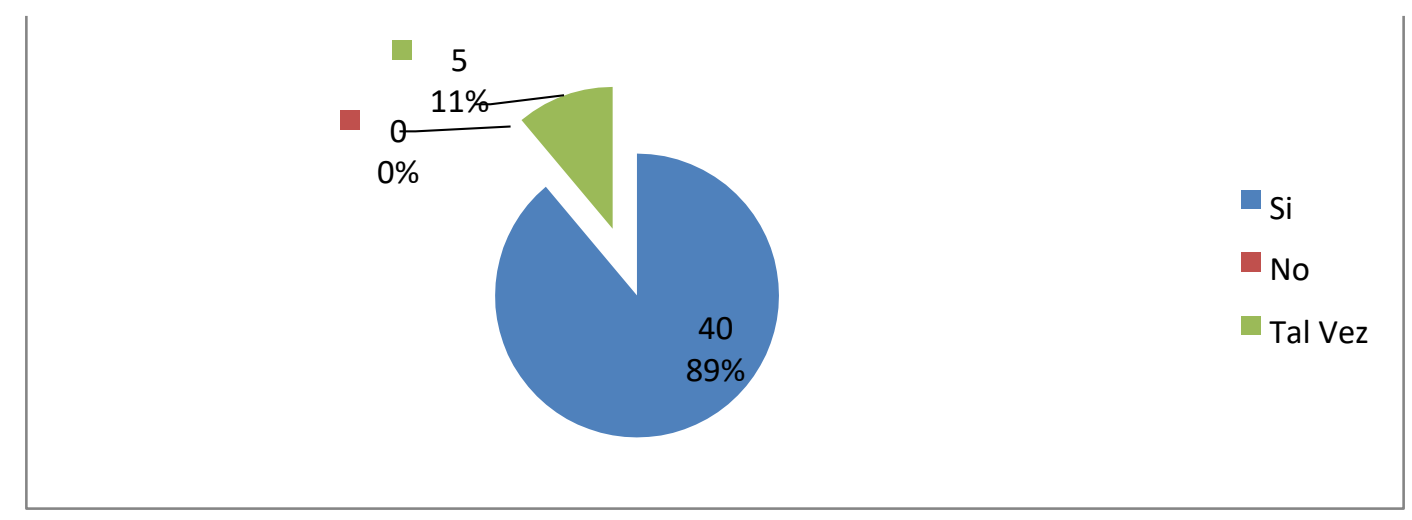

Datos: Centro de Educación Básica $N^{\circ} 508$ “Dr. Clemente Yerovi Indaburu” 


\section{Análisis e Interpretación}

En esta interrogante el $89 \%$ de los estudiantes encuestados respondieron que les ayudaba a aprender Lengua y Literatura trabajando con organizadores gráficos; el 11\% respondió tal vez y nadie respondió no.

Analizando las respuestas obtenidas se llega a la conclusión que a los estudiantes les ayuda mucho trabajar con organizadores gráficos, que es una técnica activa que los motiva a un aprendizaje fácil del área Lengua y Literatura, además es una de las mejores técnicas hará enseñar las habilidades del pensamiento.

\section{5.- ¿Todos los trabajos que realizan en clase lo hacen en equipo?}

\section{CUADRO $\mathbf{N}^{\circ} 13$}

\begin{tabular}{|l|l|c|c|}
\hline $\mathbf{N}^{\circ}$ & INDICADORES & FRECUENCIA & $\boldsymbol{\%}$ \\
\hline $\mathbf{1}$ & SI & 6 & 13 \\
\hline $\mathbf{2}$ & NO & 32 & 71 \\
\hline $\mathbf{3}$ & A VECES & 7 & 16 \\
\hline & TOTAL & 45 & 100 \\
\hline
\end{tabular}

Datos: Centro de Educación Básica $N^{\circ} 508$ “Dr. Clemente Yerovi Indaburu” 


\section{GRÁFICO $\mathbf{N}^{\circ} 13$}
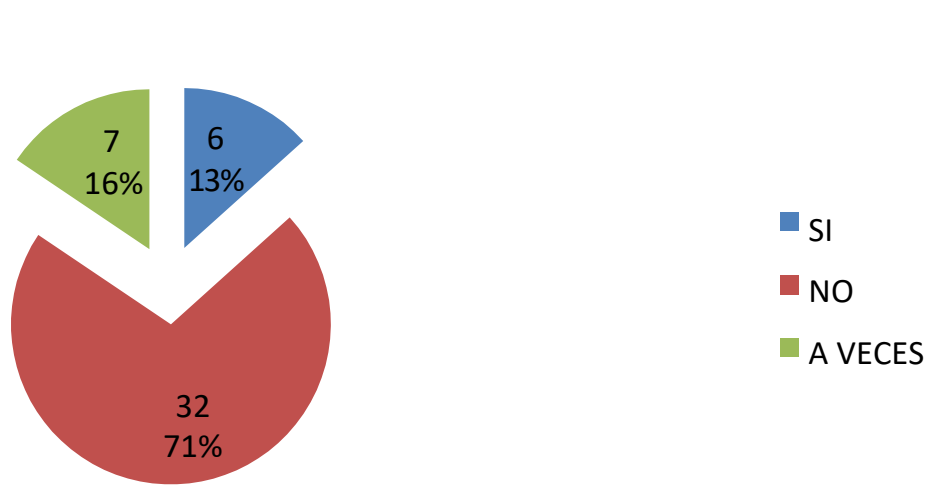

Datos: Centro de Educación Básica No 508 “Dr. Clemente Yerovi Indaburu”

\section{Análisis e Interpretación}

El $71 \%$ de los estudiantes encuestados respondieron que los trabajos que se realizan en clase no los hacen en equipo; el 16\% respondieron que a veces y el 13\% que si lo realizan en equipo.

Analizando estas respuestas podemos deducir que la maestra no realiza la participación de los estudiantes en el aula por lo que no hay interacción de aprendizaje y su metodología es tradicional, con esto crea un ambiente desmotivado en grupo, creando en los estudiantes un esquema mental individualista. Las respuestas de los docentes confirman los resultados de esta pregunta a los estudiantes.

\section{Conclusiones.}

El 100\% de los docentes piensan que las técnicas activas son procesos didácticos, con estos resultados se establece la importancia que tiene su utilización para el aprendizaje de los estudiantes. 
El 44\% d los docentes piensan que los aspectos que se deben tener en cuenta al momento de utilizar una técnica, son las actitudes del grupo y sus necesidades.

El 50\% de los docentes no utilizan las técnicas activas en proceso enseñanza aprendizaje; aún, cuando están conscientes que es un recurso importante en el aprendizaje de los estudiantes.

El $75 \%$ de los docentes considera que el uso adecuado de técnicas activas en el aprendizaje del área de Lengua y Literatura promueven un rendimiento excelente en los estudiantes; pero, sin embargo, no lo aplican.

A pesar de existir varias técnicas para propiciar un aprendizaje activo en el aula, los docentes no las utilizan y solo se limitan a que los estudiantes los escuchen y escriban exactamente los conceptos que ellos les dictan.

Los docentes no conocen todas las técnicas que activen el desarrollo de las habilidades y destrezas de los estudiantes en el área de Lengua y Literatura.

Las destrezas que más fácil se les hace a los docentes desarrollar en los estudiantes durante el proceso aprendizaje es: Escuchar y escribir, aunque piensan que hay otras importantes, pero no saben cómo hacer que los estudiantes las desarrollen.

El $62 \%$ de los estudiantes piensan que el trabajo que realiza su maestra en el aula es tedioso para ellos, por lo que se determina que no utilizan las técnicas didácticas activas

Con respecto a los estudiantes respondieron que los trabajos que se realizan en clase no los hacen en equipo; sino, individualmente. 
Janet E. Pazmiño-Ramírez; Silvia A. Medina-Anchundia; Juan C. Ramos-Romero

En el Centro de Educación Básica "Dr. Clemente Yerovi Indaburu no existe una guía didáctica que los ayude para que puedan aplicarlas en sus clases.

\section{Recomendaciones.}

Es importante que el Director de la institución tome en consideración los resultados de la presente investigación a fin de implementar estrategias que permitan capacitar a los docentes del establecimiento sobre la importancia que tienen las técnicas activas para el aprendizaje del área de Lengua y Literatura.

Que los docentes inicien con una motivación al principio de una hora clases ya que de esta manera obtendrán el interés y atención de los estudiantes durante el desarrollo de la clase.

Que los docentes promuevan la participación activa de los estudiantes, aplicando diversas actividades creando un ambiente de confianza en ellos para que expresen sus experiencias y conocimientos espontáneamente y sin temor a ser rechazados.

Se sugiere a los a los maestros utilizar las técnicas adecuadas que se relacione con sus contenidos para que el proceso de interaprendizaje sea utilizado en una forma activa y dinámica.

Se propone al docente emplear en sus aulas de clase; las distintas técnicas grupales existentes ya que mediante esta forma de trabajo los niños que tienen un poco de problema en intervenir individualmente, lo harán con entusiasmo y mayor frecuencia, además de ser ellos mismos que construyan sus aprendizajes.

Que los profesores tengan un espíritu de cambio y flexibilidad y flexibilidad, si una técnica no les da resultado cambiarla inmediatamente por otra. 
Se aconseja aplicar correctamente las técnicas conocidas en su labor diaria como docente, tomándolas como un modo fundamental actual de poder lograr la atención y motivación de los estudiantes.

Se plantea que los docentes tengan innovación permanente, en cuanto a contenidos y técnicas activas ya sea asistiendo frecuentemente a talleres prácticos, charlas, seminario, ya que la educación se encuentra en constante cambio.

Propiciar el aprendizaje y la enseñanza conforme a las necesidades y a las expectativas que tienen los estudiantes, mediante técnicas y juegos para crear un ambiente agradable en todo el proceso de adquisición del conocimiento en el campo de Lengua y Literatura.

Se recomienda a los docentes utilizar la Guía didáctica con técnicas activas que han sido seleccionadas para el aprendizaje del área de Lengua y Literatura que será entregada al Director de la institución, además de buscar los medios idóneos, para desarrollar adecuadamente; las destrezas, habilidades y valores en los estudiantes ya que de ello depende su formación como individuos, involucrados en el desarrollo de la sociedad.

\section{Bibliografía.}

AGUERA ESPEJO-SAAVEDRA, Isabel, (2007). Ideas Prácticas para un Currículo Creativo, Edit. NARCEA, Madrid.

ÁLVAREZ.GAYOU, Jurgenson, (2008). Cómo hacer investigación cualitativa. Fundamentos y metodología. ARMIJOS REYES, Carlos y otros (2005), Investigación del Proceso Educativo, Evento 8, Edit. UNL, Loja. ASAMBLEA NACIONAL DEL ECUADOR, (2011). Ley Orgánica de

Educación Intercultural Bilingüe. Quito.

CENAISE, (2006), Tiempo de Educar, Revista del pensamiento pedagógico ecuatoriano, $\mathrm{N}^{\circ} 12$, Edit. Libresa, Quito. 
Janet E. Pazmiño-Ramírez; Silvia A. Medina-Anchundia; Juan C. Ramos-Romero

CIBOTTI, Ema (2005), Una introducción a la enseñanza de la historia latinoamericana. Buenos Aires.

Diccionario OCÉANO Manual para docentes. Didáctica General N 5. 2006.

Diccionario OCÉANO, (2006). Manual para docentes Didáctica General Nº 5 .

DICCIONARIO OCÉANO, (2011)

FLORES OCHOA, Rafael, (2006). Hacia una Pedagogía del

Conocimiento, Edit. Mc Graw Hill, Bogotá.

Guía para docentes: Lengua y Literatura Sexto y Séptimo de Básica: 2007.

HERNÁNDEZ, Juanita (2005), Estrategias Educativas para el Aprendizaje Activo, Programa de Capacitación en Liderazgo Educativo, Edit. EB-PRODEC, Quito.

MINISTERIO DE EDUCACIÓN (2009) Guía del Docente de

Lenguaje y Comunicación. Ediciones EDINUN. Quito - Ecuador.

MINISTERIO DE EDUCACIÓN DEL ECUADOR, (2010).

Actualización y Fortalecimiento Curricular de la Educación General Básica. Quito.

MINISTERIO DE EDUCACIÓN DEL ECUADOR, (2010). Lengua y

Literatura, Guía para el docente Sexto Año de Básica.

OCÉANO EDITORES, (2007), Aprender a Aprender, Edit. Grupo Océano, Barcelona.

PÉREZ Gastón y otros, (2009), Metodología de la Investigación Educacional Primera Parte, Edit. Pueblo y Educación. Cuba CP 11300.

PÍO ASASTRUÉ y otros (2007), El desarrollo de competencias en lenguas: textos y otras estrategias. Madrid: Ministerio de Educación y Ciencia. Subdirección General de Información y Publicaciones.

SILVA, Floresmilo (2006) Innovación Pedagógica Editorial Pedagógica Freire Riobamba - Ecuador.

VILLARROEL, Cesar (2009) Orientaciones Didácticas para el Trabajo Docente Impresión Oseas - Espín L. Quito - Ecuador.

ZABALA, Antoni, ARNAU, Laia (2007); Cómo aprender y enseñar competencias: 11 ideas clave, Barcelona: Editorial Grao. 